\title{
Fermi Surface Structure and Isotropic Stability of Fulde-Ferrell-Larkin-Ovchinnikov Phase in Layered Organic Superconductor $\beta^{\prime \prime}-(\mathrm{BEDT}-\mathrm{TTF})_{2} \mathrm{SF}_{5} \mathrm{CH}_{2} \mathrm{CF}_{2} \mathrm{SO}_{3}$
}

\author{
Shiori Sugiura ${ }^{1, *,+}\left(\mathbb{D}\right.$, Hiroki Akutsu $^{2}\left(\mathbb{D}\right.$, Yasuhiro Nakazawa ${ }^{2}$, Taichi Terashima ${ }^{3}\left(\mathbb{D}\right.$, Syuma Yasuzuka $^{4}$, \\ John A. Schlueter ${ }^{5,6}$ (D) and and Shinya Uji ${ }^{3, *,+}$ \\ 1 Institute for Materials Research, Tohoku University, Sendai 980-8577, Japan \\ 2 Department of Chemistry, Graduate School of Science, Osaka University, Toyonaka 560-0043, Japan; \\ akutsu@chem.sci.osaka-u.ac.jp (H.A.); nakazawa@chem.sci.osaka-u.ac.jp (Y.N.) \\ 3 Institute for Materials Science, Tsukuba 305-0003, Japan; TERASHIMA.Taichi@nims.go.jp \\ 4 Research Center for Condensed Matter Physics, Hiroshima Institute of Technology, \\ Hiroshima 731-5193, Japan; yasuzuka@cc.it-hiroshima.ac.jp \\ 5 Materials Science Division, Argonne National Laboratory, Argonne, IL 60439, USA; JASchlueter@anl.gov \\ 6 Division of Materials Research, National Science Foundation, Alexandria, VA 22314, USA \\ * Correspondence: shiori.sugiura.c5@tohoku.ac.jp (S.S.); UJI.shinya@nims.go.jp (S.U.); \\ Tel.:+81-22-215-2028 (S.S.); +81-29-863-5512 (S.U.) \\ + These authors contributed equally to this work.
}

\section{check for} updates

Citation: Sugiura, S.; Akutsu, H.; Nakazawa, Y.; Terashima, T. Yasuzuka, S.; Schlueter, J.A.; Uji, S. Fermi Surface Structure and Isotropic Stability of Fulde-Ferrell-LarkinOvchinnikov Phase in Layered Organic Superconductor $\beta^{\prime \prime}$-(BEDT-TTF) ${ }_{2} \mathrm{SF}_{5} \mathrm{CH}_{2} \mathrm{CF}_{2} \mathrm{SO}_{3}$. Crystals 2021, 11, 1525. https:// doi.org/10.3390/cryst11121525

Academic Editor: Andrej Pustogow

Received: 20 November 2021

Accepted: 1 December 2021

Published: 7 December 2021

Publisher's Note: MDPI stays neutral with regard to jurisdictional claims in published maps and institutional affiliations.

Copyright: (c) 2021 by the authors. Licensee MDPI, Basel, Switzerland. This article is an open access article distributed under the terms and conditions of the Creative Commons Attribution (CC BY) license (https:/ / creativecommons.org/licenses/by/ $4.0 /)$.

\begin{abstract}
The Fermi surface structure of a layered organic superconductor $\beta^{\prime \prime}$-(BEDT-TTF) ${ }_{2} \mathrm{SF}_{5} \mathrm{CH}_{2} \mathrm{CF}_{2} \mathrm{SO}_{3}$ was determined by angular-dependent magnetoresistance oscillations measurements and band-structure calculations. This salt was found to have two small pockets with the same area: a deformed square hole pocket and an elliptic electron pocket. Characteristic corrugations in the field dependence of the interlayer resistance in the superconducting phase were observed at any in-plane field directions. The features were ascribed to the commensurability (CM) effect between the Josephson vortex lattice and the periodic nodal structure of the superconducting gap in the Fulde-Ferrell-Larkin-Ovchinnikov (FFLO) phase. The CM effect was observed in a similar field region for various in-plane field directions, in spite of the anisotropic nature of the Fermi surface. The results clearly showed that the FFLO phase stability is insensitive to the in-plane field directions.
\end{abstract}

Keywords: organic superconductor; resistance; FFLO phase; vortex dynamics

\section{Introduction}

The discovery of superconductivity has led to breakthroughs in a wide range of fields from fundamental research and applications [1]. In particular, since the discovery of high-temperature superconducting cuprates in 1980s, the search for new superconducting mechanisms has been one of the major trends in superconductivity basic research. Among the various superconductors, organic superconductors in the vicinity of metal-insulator transitions have brought about significant progress in basic research.

Organic conductors based on BEDT-TTF molecules are characterized by a stacked structure with anion molecule (insulating) layers and BEDT-TTF molecule (conducting) layers. These conductors have attracted significant interest because of the presence of various ground states, a dimer-Mott insulating phase, a charge-ordered phase, a density wave phase, and a superconducting phase, where the degree of dimerization of the BEDT-TTF molecules, the Fermi surface instability, and the strong electron correlation play important roles. In particular, the possibility of unconventional superconductivity, mediated by antiferromagnetic spin and/or charge fluctuations, is a central concern.

When the orbital effect is suppressed and the critical field $\left(H_{\mathrm{c} 2}\right)$ is Pauli-limited, a unique superconducting phase, namely, the Fulde-Ferrell-Larkin-Ovchinnikov (FFLO) 
superconducting phase is expected to emerge at high fields [2,3]. In conventional superconductors, the spin-singlet Cooper pairs formed by up and down spins are destroyed in a magnetic field by the Zeeman effect. This pair-breaking effect gives the Pauli limit, $H_{\text {Pauli }}=\Delta_{0} / \sqrt{2} \mu_{\mathrm{B}}=1.86 T_{\mathrm{C}}$, where $\Delta_{0}$ is the superconducting energy gap at $0 \mathrm{~K}$, and $\mu_{\mathrm{B}}$ is the Bohr magneton [4]. In the FFLO phase, the Cooper pairs are formed between up and down spins on the polarized Fermi surface. Therefore, the Cooper pairs have a finite center-of-mass momentum $q$ and show a spatial modulation of the order parameter in real space; $\Delta(\boldsymbol{r})=\Delta \cos (\boldsymbol{q} r)$, as shown in Figure 1a. As a result, the superconductivity can be stabilized even above $H_{\text {Pauli }}$. In recent years, experimental results suggesting its existence have been obtained in heavy fermion superconductors $[5,6]$, oxide layered superconductors [7], ion-based superconductors [8], and organic superconductors [9-16]. In organic superconductors, the FFLO phase transition was first observed by a tuned-circuit differential susceptometer experiment for $\kappa-(\mathrm{BEDT}-\mathrm{TTF})_{2} \mathrm{Cu}(\mathrm{NCS})_{2}$ [10], and, since then, various measurements [17-24] have been performed to confirm the FFLO transition.

(a)

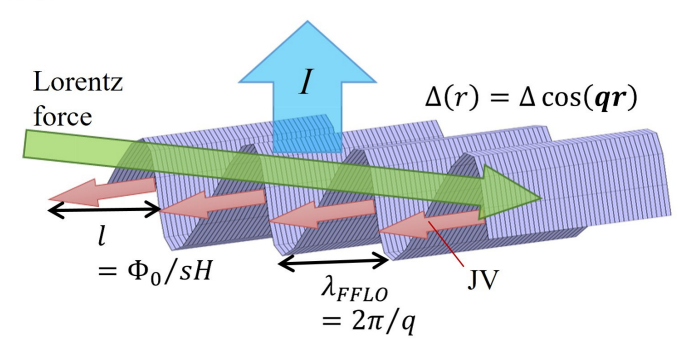

(b)

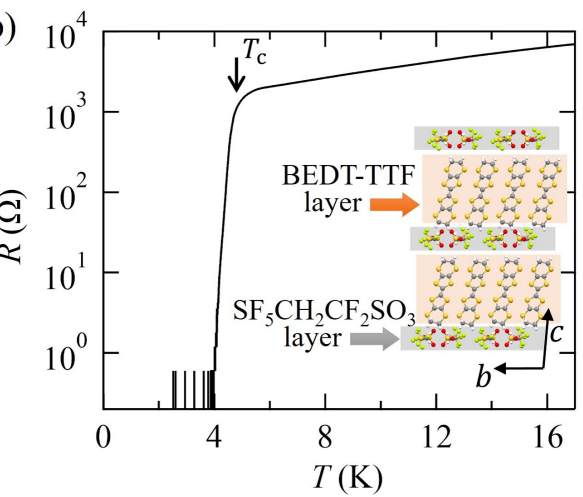

Figure 1. (a) Schematic illustration of the order parameter oscillation $\Delta(\boldsymbol{r})$ for a single $\boldsymbol{q}$ case in a FFLO phase and JV lattice in a layered superconductor. The JVs are easily driven by the Lorentz force in an interlayer current $I$, leading to nonzero interlayer resistance even in the superconducting phase. (b) Temperature dependence of the interlayer resistance of the $\beta^{\prime \prime}-\mathrm{SF}_{5}$ salt. The onset of the superconducting transition can be defined as $T_{\mathrm{c}} \approx 4.8 \mathrm{~K}$, consistent with the specific heat measurement [23]. Inset: crystal structure of $\beta^{\prime \prime}-\mathrm{SF}_{5}$ salt.

Highly two-dimensional (2D) layered superconductors can be modeled as Josephsoncoupled multi-layer systems. In such superconductors, magnetic flux lines penetrating the sample can be decomposed into two parts; the pancake vortices (PVs) penetrating the superconducting layers and the Josephson vortices (JVs) penetrating the insulating layers. The JVs are pinned more loosely than the PVs, since the order parameter vanishes in the insulating layers. Therefore, the JVs are easily driven by the Lorentz force in an interlayer current, and, consequently, nonzero interlayer resistance is observed even in the superconducting phase. In the FFLO phase, periodic nodal lines of the order parameter are formed by the finite center-of-mass momentum $q$ of the Cooper pairs as depicted in Figure 1a. When the nodal lines are parallel to the JVs, they will work as pinning sites of the JVs. The wavelength of the order parameter oscillation is given by $\lambda_{\mathrm{FFLO}}=2 \pi / q$, and the JV lattice spacing is $l=\Phi_{0} / s H$, where $s$ is the interlayer spacing and $\Phi_{0}$ is the flux quantum. It is expected that JVs are relatively strongly pinned by the nodal line structure for a commensurate condition $l / \lambda_{\text {FFLO }}=N\left(N\right.$ : integer). Since $\lambda_{\text {FFLO }}$ is also expected to decrease with an increasing field $[25,26]$, the commensurate condition in the FFLO phase will be periodically satisfied, leading to fine structures in the interlayer resistance curves. This commensurability (CM) effect was first predicted by Bulaevskii et al. [27]. Thus far, the CM effect has been observed in the FFLO phases for various organic superconductors, $\lambda$-(BETS) ${ }_{2} \mathrm{FeCl}_{4}$ [15], $\beta^{\prime \prime}-(\mathrm{BEDT}-\mathrm{TTF})_{4}\left[\left(\mathrm{H}_{3} \mathrm{O}\right) \mathrm{Ga}\left(\mathrm{C}_{2} \mathrm{O}_{4}\right)_{3}\right] \mathrm{C}_{6} \mathrm{H}_{5} \mathrm{NO}_{2}$ ( $\beta^{\prime \prime}$-Ga salt) [28], and $\beta^{\prime \prime}-(\mathrm{BEDT}-\mathrm{TTF})_{2} \mathrm{SF}_{5} \mathrm{CH}_{2} \mathrm{CF}_{2} \mathrm{SO}_{3}\left(\beta^{\prime \prime}-\mathrm{SF}_{5}\right.$ salt) [29]. Among them, the highly $2 \mathrm{D}$ nature of 
the $\beta^{\prime \prime}$-Ga and $\beta^{\prime \prime}-\mathrm{SF}_{5}$ salts with the large anion layers would provide an excellent platform for the FFLO studies, since JV dynamics play an essential role in the CM effects.

The $\beta^{\prime \prime}-\mathrm{SF}_{5}$ salt with $T_{\mathrm{c}} \approx 4.8 \mathrm{~K}$ is composed of the conducting BEDT-TTF molecular layer and the large insulating $\mathrm{SF}_{5} \mathrm{CH}_{2} \mathrm{CF}_{2} \mathrm{SO}_{3}$ layer (inset of Figure $1 \mathrm{~b}$ ). A highly $2 \mathrm{D}$ electronic state has been realized, which is characterized by a large ratio of the intralayer to that of interlayer critical fields $H_{\mathrm{c} 2}^{\|} / H_{\mathrm{c} 2}^{\perp} \approx 11.5$. The specific-heat measurements show strongly coupled BCS-like behavior with a full gap given by $\Delta_{0} / k_{\mathrm{B}} T=2.18$ [30]. In a magnetic field parallel to the conducting layers, the critical field $H_{\mathrm{c} 2}$ significantly exceeded the Pauli limit $H_{\text {Pauli }} \approx 10 \mathrm{~T}$, above which the FFLO superconductivity is realized [31-35]. Optical measurements of isostructural $\beta^{\prime \prime}$-(BEDT-TTF) $)_{2} \mathrm{SF}_{5} \mathrm{RSO}_{3}\left(R=\mathrm{CH}_{2}, \mathrm{CHFCF}_{2}, \mathrm{CH}_{2} \mathrm{CF}_{2}\right.$, and $\mathrm{CHF}$ ) compounds revealed that the superconducting phase is adjacent to a chargeordered insulating phase [36]. This could indicate superconductivity mediated by charge fluctuations, which is another reason for the interest in the $\beta^{\prime \prime}$ salts.

In our previous studies, we clarified the FFLO phase boundary in terms of the magnetocaloric effect, torque [35], and resistance measurements [29]. We also observed the CM effect in fields almost parallel to the $a$-axis in the FFLO phase, above $\sim 9 \mathrm{~T}$. The $\lambda_{\text {FFLO }}$ values, ranging from $\sim 40 \mathrm{~nm}$ to $\sim 210 \mathrm{~nm}$, were obtained under the assumption of a single $q$ vector perpendicular to the field. The stability of the FFLO phase is closely related to the nesting instability of the Fermi surface, and the $q$ vector leading to a large nesting part is favorable for the FFLO phase. Therefore, the optimum $q$ vector depends on the anisotropic structure of the Fermi surface. Meanwhile, the orbital effect stabilizes the $\boldsymbol{q}$ vector parallel to the field. This situation can lead to complicated field-direction dependencies of the optimum $\boldsymbol{q}$ vector. Even multi- $\boldsymbol{q}$-vector phases are theoretically predicted depending on the field strength and temperature [37].

In this study, we focused on the stability of the FFLO phase in the $\beta^{\prime \prime}-\mathrm{SF}_{5}$ salt, with an anisotropic Fermi surface. Firstly, we clarify the Fermi surface structure from the measurements of angular-dependent magnetoresistance oscillations (AMROs), and then we report the $\mathrm{CM}$ effect in various in-plane field directions. The $\mathrm{CM}$ effect was surprisingly observed in a similar field region for various in-plane field directions, despite the anisotropic Fermi surface structure. Possible scenarios for explaining these results are presented.

\section{Materials and Methods}

Single crystals of the $\beta^{\prime \prime}-\mathrm{SF}_{5}$ salt were synthesized using a standard electrochemical method [38]. Two gold wires of $10 \mu \mathrm{m}$ diameter were attached to both sides of the single crystal using carbon paste. The interlayer resistance with an electric current perpendicular to the superconducting layers was measured using a conventional four-probe AC technique. The single crystals were mounted on a two-axis rotator in a ${ }^{3} \mathrm{He}$ cryostat with a $15 \mathrm{~T}$ superconducting magnet and cooled down to $\sim 0.5 \mathrm{~K}$ at a rate of $\sim 1 \mathrm{~K} / \mathrm{min}$. All measurements were performed at the Tsukuba Magnet Laboratories, NIMS.

\section{Results}

Figure $1 \mathrm{~b}$ shows the temperature dependence of the interlayer resistance for the $\beta^{\prime \prime}-\mathrm{SF}_{5}$ salt. The resistance decreased monotonically with decreasing temperature. At $\sim 4.8 \mathrm{~K}$, a sudden drop in resistance was observed due to the superconducting transition. Below $\sim 4 \mathrm{~K}$, the resistance was zero within the noise level. To investigate the 2D Fermi surface structure, we first measured the AMROs in various rotation planes. Typical AMRO data are presented in Figure 2a. The angles $\theta$ and $\varphi$ are defined in the inset. The characteristic $\theta$ dependence of the interlayer resistance is shown in the upper part of Figure 2a. In the negative second derivative curves, we can observe AMROs, which are periodic with $\tan (\theta)$, as shown in the lower part of Figure 2a. The AMRO period $\delta$ directly yields the reciprocal lattice vector $\boldsymbol{k}_{\|}, \delta(\boldsymbol{r})=\pi / s \boldsymbol{k}_{\|}(\varphi)$ values. Figure $2 \mathrm{~b}$ shows the polar plot of $\boldsymbol{k}_{\|}$ obtained from the AMRO measurements at various $\varphi$. We can draw the cross-section of the 2D Fermi surface, inscribed in the $\boldsymbol{k}_{\|}(\varphi)$ curves, by a solid curve, assuming an elliptical shape. The cross-section of the Fermi surface is very elongated, whose area was $\sim 6 \%$ of 
the first Brillouin zone. The AMRO results were almost consistent with those of previous reports [39].
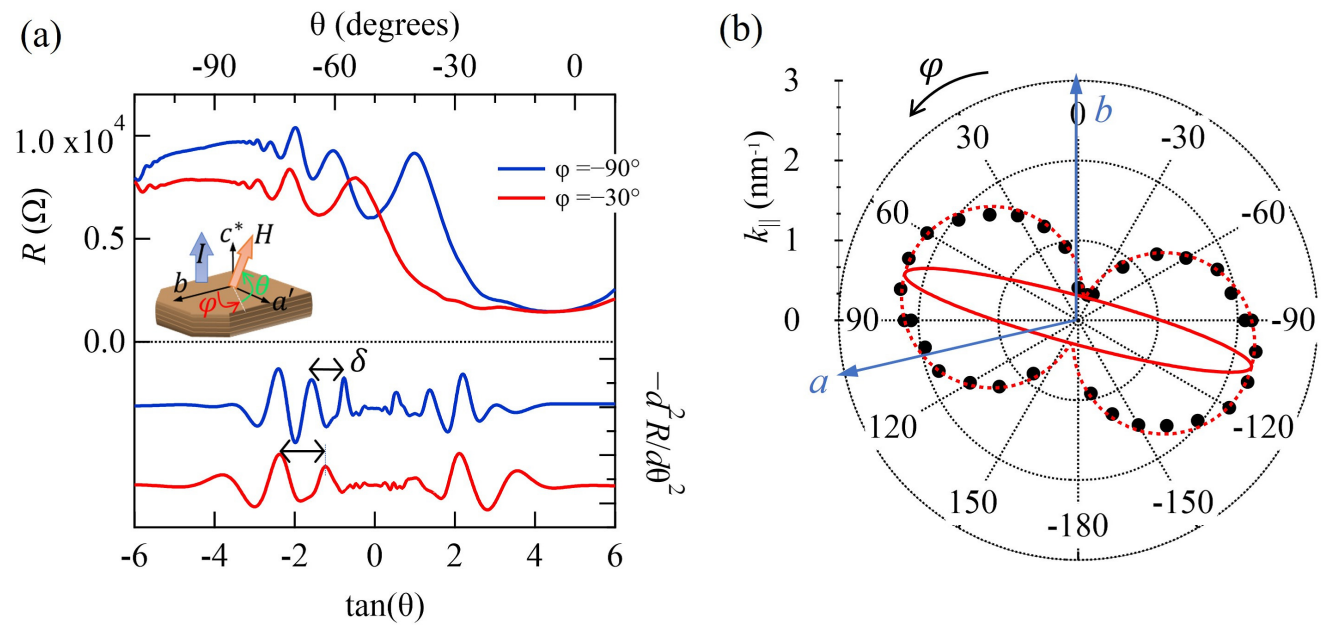

Figure 2. (a) Typical AMRO data and their negative second derivative curves at $1.5 \mathrm{~K}$ for $14 \mathrm{~T}$. (b) Polar plot of $\boldsymbol{k}_{\|}$obtained from the AMRO measurements. The red solid curve shows the 2D Fermi surface obtained from the AMRO measurements.

In Figure 3a, we present the band calculations by an extended Huckel method [40], using lattice parameters obtained from X-ray crystallography [38]. The calculated 2D Fermi surface is depicted in Figure $3 b$. The results are different from the reported Fermi surface structure, with a pair of 1D Fermi surface and a closed Fermi surface [38,41], in which the Brillouin zone is apparently wrong. In our calculations, there were two pockets with different carriers: a deformed square electron pocket and an elliptic hole pocket. The areas were equal to each other, and a compensated metal was formed. This is consistent with a single frequency of the quantum oscillation [42-44]. For comparison, the Fermi surface obtained from the AMRO measurements is indicated by a red dotted curve, which is almost consistent with the electron pocket. In the AMRO measurements, the hole pocket was not observed. The reason for this is not clear at present.

(a)

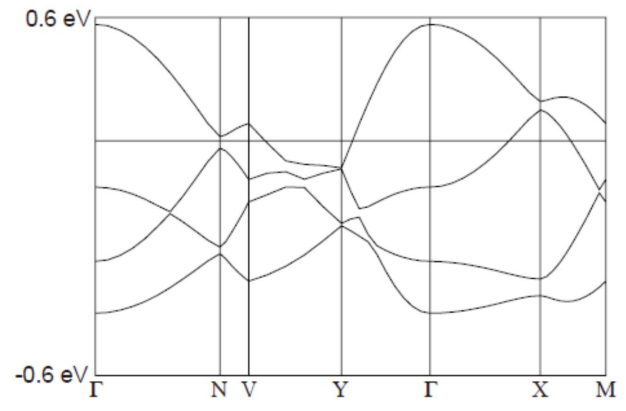

(b)

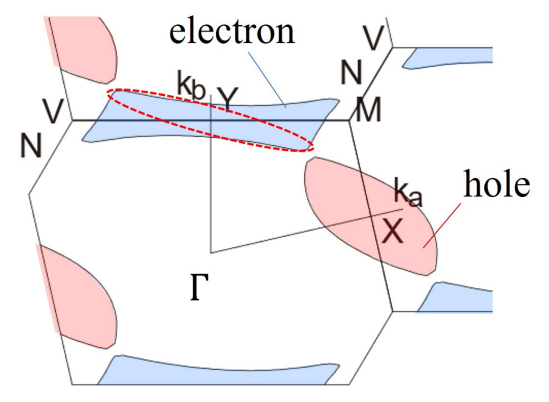

Figure 3. (a) Band structure by an extended Huckel method and (b) 2D Fermi surface structure for the $\beta^{\prime \prime}-\mathrm{SF}_{5}$ salt. Deformed square hole and elliptic electron pockets were formed. The red dotted curve indicates the 2D Fermi surface determined by the AMRO measurements.

Figure 4a shows the magnetic field dependence of the interlayer resistance at various temperatures. The field was applied parallel to the $b$ axis, in the superconducting $a^{\prime}-b$ plane, within the accuracy of $0.1^{\circ}$. At $0.5 \mathrm{~K}$, the resistance increased with the field above $6.5 \mathrm{~T}$, defined as $H_{\text {onset }}$. Characteristic corrugation was evident. The corrugation was reduced with increasing temperature. The critical field was determined as $H_{\mathrm{c} 2} \approx 13 \mathrm{~T}$ at 0.7 K from the specific-heat measurements [23]. The finite resistance in the wide field region 
below $H_{\mathrm{c} 2}$ can be ascribed to the motion of the JVs in the insulating layers, as has been observed in various organic superconductors $[15,28,29]$. To clearly see the corrugations in detail, negative second-derivative curves of the resistance are plotted in Figure $4 \mathrm{~b}$. At $0.5 \mathrm{~K}$, we see a broad dip at $\sim 6.5 \mathrm{~T}$, corresponding to the resistance increase from the noise level. Above $\sim 8 \mathrm{~T}$, we see a quasi-periodic dip structure, which is most pronounced at $\sim 10 \mathrm{~T}$. This structure can be ascribed to the CM effect, which is observable only in the FFLO phase, as discussed in the previous reports [29]. At higher fields, the CM effect is reduced and vanishes above $\sim 12 \mathrm{~T}$, which corresponds to the melting transition of the JV lattice. As temperature increases, the dip structure is suppressed and shifts to a lower field region. Above $2.1 \mathrm{~K}$, such astructure is not evident. As has been discussed [27,29], the dips mean relatively strong pinning of the JV lattice, ascribed to the $\mathrm{CM}$ effect between the JV lattice and the periodic nodal structure of the gap $\Delta(\boldsymbol{r})$.
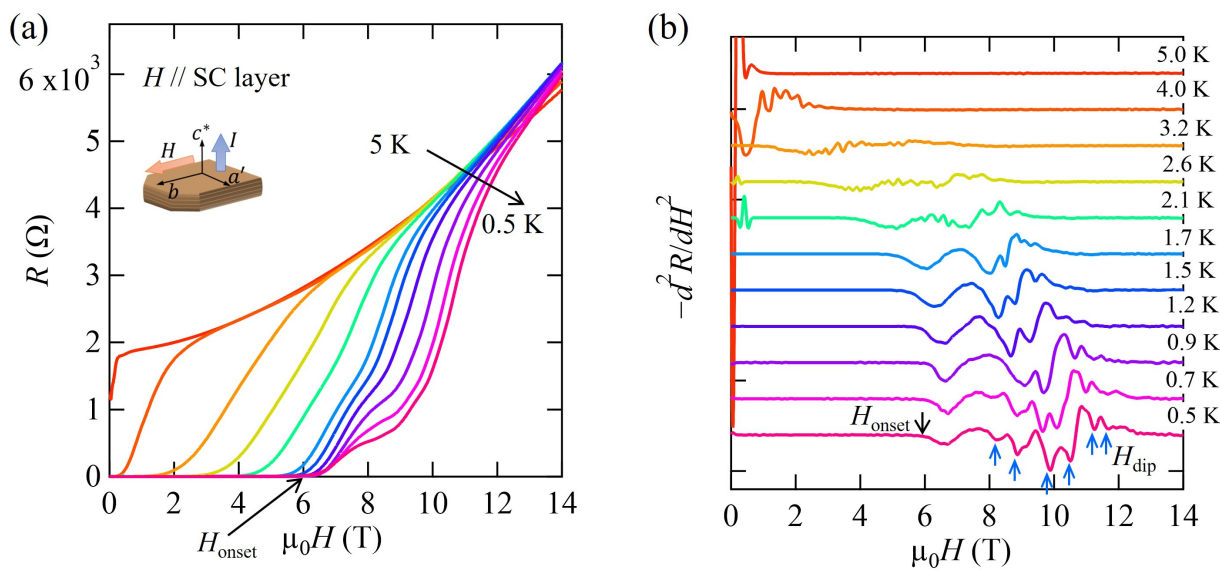

Figure 4. (a) Magnetic field dependence of the interlayer resistance at various temperatures. The field was applied parallel to the $b$ axis in the superconducting $a^{\prime}-b$ plane within an accuracy of $0.1^{\circ}$. (b) Negative second-derivative curves of the resistance. Each curve was shifted for clarity.

From the above results, we obtained the temperature-field phase diagram shown in Figure 5. The blue squares indicate the dip fields $H_{\text {dip }}$ and the solid curve indicates $H_{\mathrm{c} 2}$, which was determined from specific-heat measurements [23]. The FFLO phase appears in a wide region above $\sim 8 \mathrm{~T}$ and below $\sim 2 \mathrm{~K}$. The phase diagram is very similar to that for the $H \| a$-axis [29], although the Fermi surface was anisotropic.

Figure 6a shows the magnetic field dependence of the resistance at various field angles $\theta$. For $\theta=0^{\circ}(H \| b$-axis $)$, the resistance increased with the field above $\mu_{0} H_{\text {onset }}=6 \mathrm{~T}$, which is indicated by an arrow. Figure $6 \mathrm{~b}$ shows the negative second-derivative curves of the resistance. The low $H_{\text {onset }}$ value for $\theta=0^{\circ}$, denoted by an arrow, indicates that only JVs were formed (no PVs), which were pinned very weakly in the insulating layers. When the field was tilted from the superconducting layer, $H_{\text {onset }}$ increased. This behavior is explained by the stronger pinning of the flux lines in the superconducting layers, where PVs are formed. As the field was further tilted, $H_{\mathrm{c} 2}$ was steeply reduced, leading to a decrease in $H_{\text {onset. }}$. For $\theta=0^{\circ}$, small dips due to the $\mathrm{CM}$ effect can be seen above $\sim 9.5 \mathrm{~T}$. As the field was tilted from the layer, the $\mathrm{CM}$ effect was suppressed, and no $\mathrm{CM}$ effect was observed for $|\theta| \gtrsim 0.6^{\circ}$. The stability of the FFLO phase in such a small angle region is consistent with the results for the field almost parallel to the $a$ axis [29]. 


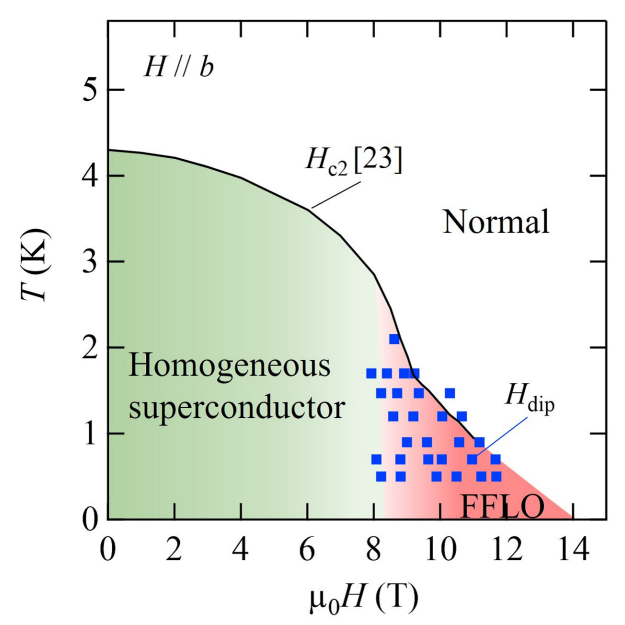

Figure 5. Temperature-field phase diagram for $H \| b$ axis. $H_{\mathrm{c} 2}$ determined by the specific-heat measurements is indicated by a solid curve [23].

(a)

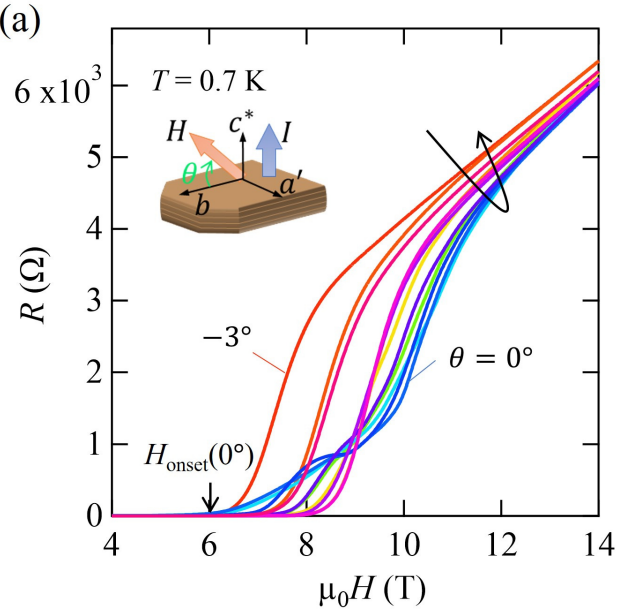

(b)

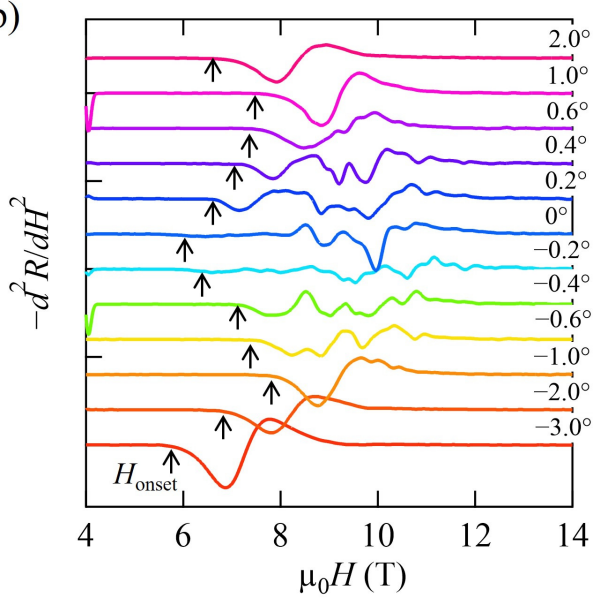

Figure 6. (a) Magnetic field dependence of the resistance at various field angles $\theta$. Definition of $\theta$ is in the inset. (b) Negative second-derivative curves of the resistance. Each curve is shifted for clarity.

Figure 7a shows the field dependence of the negative second-derivative curves at various in-plane field directions $\varphi$, as shown in the inset of Figure $7 \mathrm{~b}$. For $\varphi=0^{\circ}$, we see the onset field $\mu_{0} H_{\text {onset }}=6 \mathrm{~T}$ (black arrow). The $H_{\text {onset }}$ value had a nonmonotonic $\varphi$ dependence. It should be noted that $H_{\text {onset }}$ is the depinning field of the JV lattice [29], determined by the pinning strength at the sample edges and/or some other (impurity or defect) pinning sites, which is not related to the FFLO phase transition. The anisotropic behavior of $H_{\text {onset }}$ is possibly due to the shape effect of the sample. In contrast, we observed many dips above $\sim 9 \mathrm{~T}$, owing to the $\mathrm{CM}$ effect in a similar field region at any $\varphi$. This suggests that the FFLO phase stability was insensitive to the in-plane field direction. An important feature is that the largest dip was evident at $\mu_{0} H_{\text {dip }}^{*} \approx 9 \mathrm{~T}$ (red arrow) in a wideangle region, except for $\varphi=0^{\circ}-45^{\circ}$. This $\varphi$ dependence of the dip amplitude suggests some differences in the JV dynamics in the FFLO phase. The largest dip field $H_{\text {dip }}^{*}$ corresponds to the strongest $\mathrm{CM}$ effect and is plotted as a function of $\varphi$ in Figure $7 \mathrm{~b}$. We note that $H_{\mathrm{dip}}^{*}$ is almost isotropic, despite the anisotropic Fermi surface structure as presented in Figure 3b. 
(a)

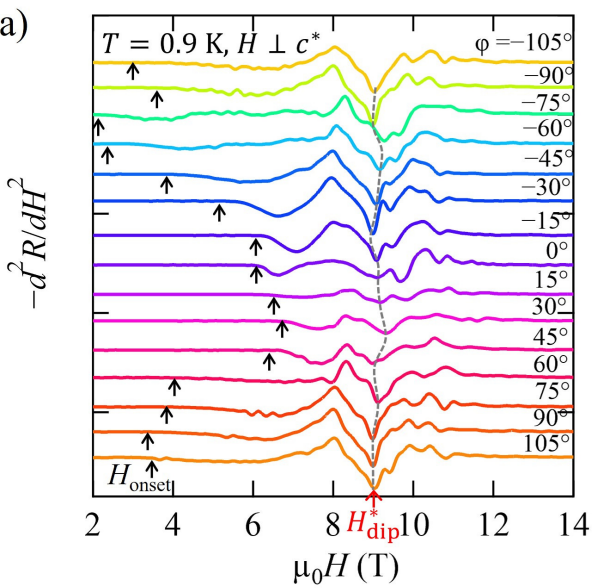

(b)

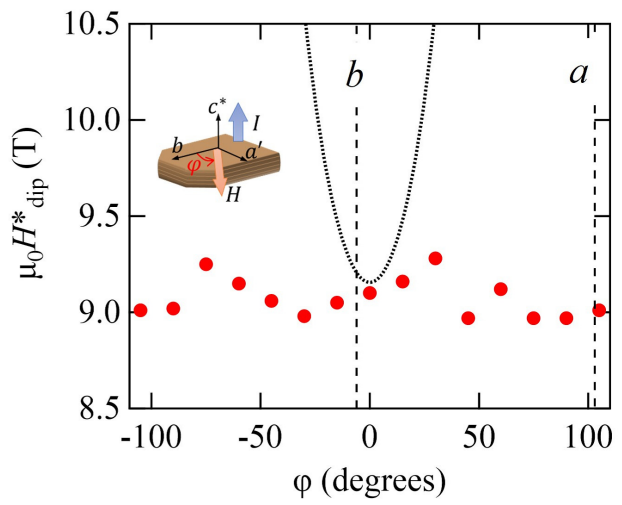

Figure 7. (a) Magnetic field dependence of the negative second-derivative curves at various in-plane field directions $\varphi$ defined in the inset of (b). Each curve is shifted for clarity. The onset field $H_{\text {onset }}$ and the largest dip field $H_{\text {dip }}^{*}$ are indicated by black and red arrows, respectively. (b) $H_{\text {dip }}$ as a function of $\varphi$. The dotted curve indicates the expected value for the CM condition $H_{\text {dip }} \propto 1 / \cos (\varphi)$ with a single $\boldsymbol{q}$ vector.

\section{Discussion}

We observed the CM effect in various in-plane field directions, which is recognized as strong evidence of the FFLO phase characterized by the $q$ vector. As pointed out, the $q$ vector leading to a large nesting part is favorable for the FFLO phase, as schematically depicted in Figure 8a, where the largest number of Cooper pairs can be formed by the $q$ vector, perpendicular to the flat part of the Fermi surface. On the other hand, in the presence of the orbital effect, the $q$ vector parallel to the field is favorable for an isotropic Fermi surface, leading to no $\mathrm{CM}$ effect. The observation of the $\mathrm{CM}$ effect at any $\varphi$ indicates that the $q$ vector is not parallel to the field.

(a)

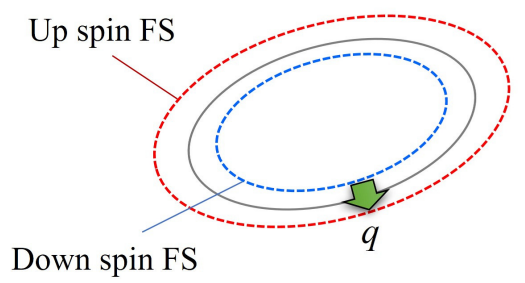

(b) $\Delta(r)=\cos (\boldsymbol{q} r)$

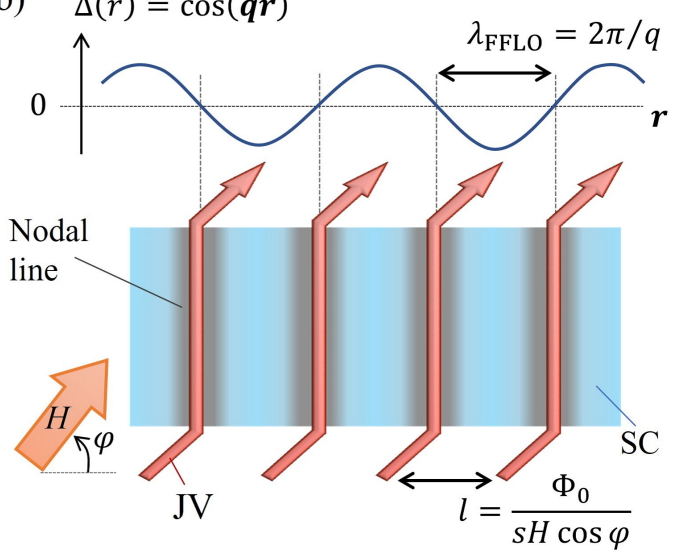

Figure 8. (a) Spin-polarized 2D Fermi surface in a magnetic field. Up and down Fermi surfaces are indicated by red and blue curves, respectively. (b) Schematic nodal line structure of a FFLO phase and JV lattice in a tilted field for the CM condition $l / \lambda_{\mathrm{FFLO}}=1$.

The largest dip at $H_{\mathrm{dip}}^{*}$ in Figure 7a suggest the strongest $\mathrm{CM}$ effect, $l / \lambda_{\mathrm{FFLO}}=1$, where all the flux lines can fit into the nodal lines completely. Assuming that the $q$ vector is fixed to a certain direction (for instance, $a^{\prime}$-axis), the JV lattice spacing is given by $l=\Phi_{0} / s H \cos (\varphi)$ as depicted in Figure $8 \mathrm{~b}$. This leads to large $\varphi$ dependence of $H_{\text {dip }}^{*}$ as indicated by the dotted curve in Figure $7 \mathrm{~b}$, which is inconsistent with the experimental result. This inconsistency requires another factor on the stability of the FFLO phase. At the nodal lines, the order 
parameter vanishes in the superconducting layers. Therefore, the most stable condition of the vortex structure will be that all the flux lines are almost parallel to the nodal lines; the $q$ vector is almost perpendicular to the field. This suggests that the direction of the $q$ vector changes with the field direction. In the anisotropic Fermi surface, the $q$ vector length should also be anisotropic, depending on the energy dispersion. Although it is difficult to know the $\varphi$ dependence of the $q$ vector, this scenario could explain the lack of significant $\varphi$ dependence of $H_{\text {dip }}^{*}$ in Figure $7 \mathrm{~b}$.

Recent specific-heat measurements show that $H_{\mathrm{c} 2}$ is the same at a few different inplane field directions in a low temperature range [23]. The fact shows that the FFLO stability is independent of $\varphi$, and seems to be consistent with our results: no significant $\varphi$ dependence of $H_{\mathrm{dip}}^{*}$. Theoretically, the in-plane anisotropies of $H_{\mathrm{c} 2}$ are led by Fermi surface structure and orbital effects in the FFLO phase [45]. Therefore, no in-plane anisotropy of $H_{\mathrm{c} 2}$ in the specific-heat measurements suggests that the orbital effect is almost negligible, and the $q$ vector is most likely pinned to an optimal direction independent of the in-plane field direction. The inconsistency with our results remains an open question.

Another possible scenario that could explain our results is that multi- $q$ vectors [37] are formed in the $\beta^{\prime \prime}-\mathrm{SF}_{5}$ salt, since the two different Fermi pockets are present as shown in Figure $3 \mathrm{~b}$. In this case, the $\boldsymbol{q}$-dependent anisotropic stability of the FFLO phase could be smeared out; the FFLO phase may appear in a similar field range, independent of the in-plane field direction. This scenario may also explain the lack of a significant in-plane anisotropy of $H_{\mathrm{dip}}^{*}$ and $H_{\mathrm{c} 2}$.

Finally, we briefly mention the results for another FFLO superconductor $\lambda$-(BETS) ${ }_{2} \mathrm{FeCl}_{4}$, which had a pair of 1D and a 2D Fermi surfaces [15]. In this salt, the CM effect was first observed in the field-induced superconducting phase. The CM effect was clearly observed for $H \| c$ but not for $H \| a$. The results show that a single $q$ vector was fixed to the $a$-axis in the whole FFLO phase. The different behavior of the CM effect between $\beta^{\prime \prime}-\mathrm{SF}_{5}$ salt and $\lambda$-(BETS) ${ }_{2} \mathrm{FeCl}_{4}$ will be closely related to the Fermi surface structure. More detailed measurements of the CM effect in other FFLO superconductors will be required to clarify the correlation between the Fermi surface structure and the $q$ vector.

\section{Conclusions}

The AMRO measurements and band-structure calculations in the $\beta^{\prime \prime}-\mathrm{SF}_{5}$ salt show that the Fermi surface is composed of two small pockets, a deformed square electron pocket, and an elliptic hole pocket, which are different from the previous report. The CM effect in the interlayer resistance was observed in a similar field region at any in-plane field directions. This indicates that the stability of the FFLO phase is almost isotropic, which is consistent with the observations of precious specific-heat measurements. Two possible scenarios are proposed: (1) a single center-of-mass momentum $q$ of the Cooper pairs, which changes with the in-plane field direction, and (2) multi- $q$ vectors, originating from the two anisotropic Fermi surfaces.

Author Contributions: S.S., T.T. and S.U. designed the experiments. S.S. and S.Y. mainly performed the resistance measurements and analyzed the data. H.A. and Y.N. performed the band calculation. J.A.S. synthesized the single crystals. S.U. supervised the project. All authors have read and agreed to the published version of the manuscript.

Funding: This work was supported by KAKENHI 17H01144 and 20K14400.

Institutional Review Board Statement: Not applicable.

Informed Consent Statement: Not applicable.

Data Availability Statement: The data that support the findings of this study are available from the corresponding author on reasonable request. 
Acknowledgments: Work at ANL was supported by U. Chicago Argonne, LLC, Operator of Argonne National Laboratory ("Argonne"). Argonne, a U.S. Department of Energy Office of Science labor s operated under Contract No. DE-AC02-06CH11357. J.A.S. acknowledges support from the Independent Research $=$ Development program while serving at the National Science Foundation.

Conflicts of Interest: The authors declare no conflict of interest.

\section{References}

1. Martucciello, N.; Giubileo, F.; Grimaldi, G.; Corato, V. Introduction to the focus on superconductivity for energy. Supercond. Sci. Technol. 2015, 28, 070201. [CrossRef]

2. Fulde, P.; Ferrell, R.A. Superconductivity in a strong spin-exchange field. Phys. Rev. 1964, 135, A550. [CrossRef]

3. Larkin, A.I.; Ovchinnikov, Y.N. Nonuniform state of superconductors. Zh. Eksp. Teor. Fiz 1964, 47, 1136.

4. Clogston, A.M. Upper limit for the critical field in hard superconductors. Phys. Rev. Lett. 1962, 9, 266. [CrossRef]

5. Radovan, H.A.; Fortune, N.A.; Murphy, T.P.; Hannahs, S.T.; Palm, E.C.; Tozer, S.W.; Hall, D. Magnetic enhancement of superconductivity from electron spin domains. Nature 2003, 425, 51-55. [CrossRef] [PubMed]

6. Bianchi, A.R.; Movshovich, C.C.; Pagliuso, P.G.; Sarrao, J.L. Possible Fulde-Ferrell-Larkin-Ovchinnikov Superconducting State in CeCoIn. Phys. Rev. Lett. 2003, 91, 187004. [CrossRef] [PubMed]

7. Kikugawa, N.; Terashima, T.; Uji, S.; Sugii, K.; Maeno, Y.; Graf, D.; Baumbach, R.; Brooks, J. Superconducting subphase in the layered perovskite ruthenate $\mathrm{Sr}_{2} \mathrm{RuO}_{4}$ in a parallel magnetic field. Phys. Rev. B 2016, 93, 184513. [CrossRef]

8. Kasahara, S.; Sato, Y.; Licciardello, S.; Čulo, M.; Arsenijević, O.S.T.; Tominaga, T.; Böker, E.J.I.; Shibauchi, T.; Wosnitza, J.; Hussey, N.E.; et al. Evidence for an Fulde-Ferrell-Larkin-Ovchinnikov State with Segmented Vortices in the BCS-BEC-Crossover Superconductor FeSe. Phys. Rev. Lett. 2020, 124, 107001. [CrossRef]

9. Yonezawa, S.; Kusaba, S.; Maeno, Y.; Auban-Senzier, P.; Pasquier, C.; Bechgaard, K.; Jérome, D. Anomalous In-Plane Anisotropy of the Onset of Superconductivity in (TMTSF) ${ }_{2} \mathrm{ClO}_{4}$. Phys. Rev. Lett. 2008, 100, 117002. [CrossRef] [PubMed]

10. Singleton, J.; Symington, J.A.; Nam, M.S.; Ardavan, A.; Kurmoo, M.; Day, P. Observation of the Fulde-Ferrell-Larkin-Ovchinnikov state in the quasi-two-dimensional organic superconductor $\kappa-(\mathrm{BEDT}-\mathrm{TTF})_{2} \mathrm{Cu}(\mathrm{NCS})_{2}(\mathrm{BEDT}-\mathrm{TTF}=$ bis (ethylene-dithio) tetrathiafulvalene). J. Phys. Condens. Matter 2000, 12, L641. [CrossRef]

11. Tanatar, M.A.; Ishiguro, T.; Tanaka, H.; Kobayashi, H. Magnetic field-temperature phase diagram of the quasi-two-dimensional organic superconductor $\lambda$-(BETS) ${ }_{2} \mathrm{GaCl}_{4}$ studied via thermal conductivity. Phys. Rev. B 2002, 66, 134503. [CrossRef]

12. Tanatar, M.A.; Ishiguro, T.; Tanaka, H.; Kobayashi, H. Superconducting phase diagram and FFLO signature in $\lambda$-(BETS) ${ }_{2} \mathrm{GaCl}_{4}$ from rf penetration depth measurements. Phys. Rev. B 2011, 83, 224507.

13. Tanatar, M.A.; Ishiguro, T.; Tanaka, H.; Kobayashi, H. Vortex Dynamics and Diamagnetic Torque Signals in Two Dimensional Organic Superconductor $\lambda$-(BETS) ${ }_{2} \mathrm{GaCl}_{4}$. J. Phys. Soc. Jpn. 2015, 84, 104709.

14. Balicas, L.; Brooks, J.S.; Storr, K.; Uji, S.; Tokumoto, M.; Tanaka, H.; Kobayashi, H.; Kobayashi, A.; Barzykin, V.; Goŕkov, L.P. Superconductivity in an organic insulator at very high magnetic fields. Phys. Rev. Lett. 2001, 87, 067002. [CrossRef] [PubMed]

15. Uji, S.; Terashima, T.; Nishimura, M.; Takahide, Y.; Konoike, T.; Enomoto, K.; Cui, H.; Kobayashi, H.; Kobayashi, A.; Tanaka, H.; et al. Vortex dynamics and the Fulde-Ferrell-Larkin-Ovchinnikov state in a magnetic-field-induced organic superconductor. Phys. Rev. Lett. 2006, 97, 157001. [CrossRef] [PubMed]

16. Uji, S.; Kodama, K.; Sugii, K.; Terashima, T.; Takahide, Y.; Kurita, N.; Tsuchiya, S.; Kimata, M.; Kobayashi, A.; Zhou, B.; et al. Magnetic torque studies on FFLO phase in magnetic-field-induced organic superconductor $\lambda$-(BETS) ${ }_{2} \mathrm{FeCl}_{4}$. Phys. Rev. B 2012, 85, 174530. [CrossRef]

17. Lortz, R.; Wang, Y.; Demuer, A.; Böttger, P.H.M.; Bergk, B.; Zwicknagl, G.; Nakazawa, Y.; Wosnitza, J. Calorimetric Evidence for a Fulde-Ferrell-Larkin-Ovchinnikov Superconducting State in the Layered Organic Superconductor $\kappa-(\mathrm{BEDT}-\mathrm{TTF})_{2} \mathrm{Cu}(\mathrm{NCS})_{2}$. Phys. Rev. Lett. 2007, 99, 187002. [CrossRef] [PubMed]

18. Bergk, B.; Demuer, A.; Sheikin, I.; Wang, Y.; Wosnitza, J.; Nakazawa, Y.; Lortz, R. Magnetic torque evidence for the FuldeFerrell-Larkin-Ovchinnikov state in the layered organic superconductor $\kappa$-(BEDT-TTF) ${ }_{2} \mathrm{Cu}(\mathrm{NCS})_{2}$. Phys. Rev. B 2011, 83, 064506. [CrossRef]

19. Wright, J.A.; Green, E.; Kuhns, P.; Reyes, A.; Brooks, J.; Schlueter, J.; Bkato, R.; Yamamoto, H.; Kobayashi, M.; Brown, S.E. Zeeman-Driven Phase Transition within the Superconducting State of $\kappa$-(BEDT-TTF) ${ }_{2} \mathrm{Cu}(\mathrm{NCS})_{2}$. Phys. Rev. Lett. 2011, 107, 087002. [CrossRef] [PubMed]

20. Agosta, C.C.; Jin, J.; Coniglio, W.A.; Smith, B.E.; Cho, K.; Stroe, I.; Martin, C. Tozer, S.W.; Murphy, T.P.; Palm, E.C.; et al Experimental and semiempirical method to determine the Pauli-limiting field in quasi-two-dimensional superconductors as applied to $\kappa$-(BEDT-TTF $)_{2} \mathrm{Cu}(\mathrm{NCS})_{2}$ : Strong evidence of a FFLO state. Phys. Rev. B 2012, 85, 214514. [CrossRef]

21. Mayaffre, H.; Krämer, S.; Horvatić, M.; Berthier, C.; Miyagawa, K.; Kanoda, K.; Mitrović, V.F. Evidence of Andreev bound states as a hallmark of the FFLO phase in $\kappa$-(BEDT-TTF) ${ }_{2} \mathrm{Cu}(\mathrm{NCS})_{2}$. Nat. Phys. 2014, 10, 928. [CrossRef]

22. Tsuchiya, S.; Yamada, J.I.; Sugii, K.; Graf, D.; Brooks, J.S.; Terashima, T.; Uji, S. Phase boundary in a superconducting state of $\kappa$-(BEDT-TTF $)_{2} \mathrm{Cu}(\mathrm{NCS})_{2}$ : Evidence of the Fulde-Ferrell-Larkin-Ovchinnikov phase. J. Phys. Soc. Jpn. 2015, $84,034703$. [CrossRef]

23. Wosnitza, J. FFLO states in layered organic superconductors. Ann. Phys. 2018, 530, 1700282. [CrossRef] 
24. Agosta, C.C.; Fortune, N.A.; Hannahs, S.T.; Gu, S.; Liang, L.; Park, J.H.; Schleuter, J.A. Calorimetric Measurements of MagneticField-Induced Inhomogeneous Superconductivity Above the Paramagnetic Limit. Phys. Rev. Lett. 2017, 118, 267001. [CrossRef]

25. Tachiki, M.; Takahashi, S.; Gegenwart, P.; Weiden, M.; Lang, M.; Geibel, C.; Steglich, F.; Modler, R.; Paulsen, C.; Ōnuki, Y. Generalized Fulde-Ferrell-Larkin-Ovchinnikov state in heavy-fermion and intermediate-valence systems. Z. Phys. Condens. Matter 1997, 100, 369-380. [CrossRef]

26. Shimahara, H. Fulde-Ferrell state in quasi-two-dimensional superconductors. Z. Phys. Condens. Matter 1994, 50, 12760. [CrossRef] [PubMed]

27. Bulaevskii, L.; Buzdin, A.; Maley, M. Intrinsic pinning of vortices as a direct probe of the nonuniform Larkin-Ovchinnikov-FuldeFerrell state in layered superconductors $\beta^{\prime \prime}-(\mathrm{BEDT}-\mathrm{TTF})_{2} \mathrm{SF}_{5} \mathrm{CH}_{2} \mathrm{CF}_{2} \mathrm{SO}_{3}$. Phys. Rev. Lett. 2003, 90, 067003. [CrossRef]

28. Uji, S.; Iida, Y.; Sugiura, S.; Isono, T.; Sugii, K.; Kikugawa, N.; Terashima, T.; Yasuzuka, S.; Akutsu, H.; Nakazawa, Y.; et al. FuldeFerrell-Larkin-Ovchinnikov superconductivity in the layered organic superconductor $\beta^{\prime \prime}-(\mathrm{BEDT}-\mathrm{TTF})_{4}\left[\left(\mathrm{H}_{3} \mathrm{O}\right) \mathrm{Ga}_{2}\left(\mathrm{C}_{2} \mathrm{O}_{4}\right)_{3}\right] \mathrm{C}_{6} \mathrm{H}_{5} \mathrm{NO}_{2}$. Phys. Rev. B 2018, 97, 144505. [CrossRef]

29. Sugiura, S.; Terashima, T.; Yasuzuka, S.; Schlueter, J.A.; Uji, S. Josephson vortex dynamics and Fulde-Ferrell-Larkin-Ovchinnikov superconductivity in the layered organic superconductor $\beta^{\prime \prime}-(\mathrm{BEDT}-\mathrm{TTF})_{2} \mathrm{SF}_{5} \mathrm{CH}_{2} \mathrm{CF}_{2} \mathrm{SO}_{3}$. Phys. Rev. B 2019, 100, 014515. [CrossRef]

30. Wanka, S.; Hagel, J.; Beckmann, D.; Wosnitza, J.; Schlueter, J.A.; Williams, J.M.; Nixon, P.G.; Winter, R.W.; Gard, G.L. Specific heat and critical fields of the organic superconductor $\beta^{\prime \prime}-(\mathrm{BEDT}-\mathrm{TTF})_{2} \mathrm{SF}_{5} \mathrm{CH}_{2} \mathrm{CF}_{2} \mathrm{SO}_{3}$. Phys. Rev. B 1998, 57, 3084. [CrossRef]

31. Cho, K.; Smith, B.E.; Coniglio, W.A.; Winter, L.E.; Agosta, C.C.; Schlueter, J.A. Upper critical field in the organic superconductor $\beta^{\prime \prime}-(\mathrm{ET})_{2} \mathrm{SF}_{5} \mathrm{CH}_{2} \mathrm{CF}_{2} \mathrm{SO}_{3}$ : Possibility of Fulde-Ferrell-Larkin-Ovchinnikov state. Phys. Rev. B 2009, 79, 220507. [CrossRef]

32. Beyer, R.; Bergk, B.; Yasin, S.; Schlueter, J.A.; Wosnitza, J. Angle-dependent evolution of the Fulde-Ferrell-Larkin-Ovchinnikov state in an organic superconductor. Phys. Rev. Lett. 2012, 109, 027003. [CrossRef]

33. Beyer, R.; Wosnitza, J. Emerging evidence for FFLO states in layered organic superconductors. Low Temp. Phys. 2013, 39, $225-231$. [CrossRef]

34. Koutroulakis, G.; Kühne, H.; Schlueter, J.A.; Wosnitza, J.; Brown, S.E. Microscopic study of the Fulde-Ferrell-Larkin-Ovchinnikov state in an all-organic superconductor. Phys. Rev. Lett. 2016, 116, 067003. [CrossRef] [PubMed]

35. Sugiura, S.; Isono, T.; Terashima, T.; Yasuzuka, S.; Schlueter, J.A.; Uji, S. Fulde-Ferrell-Larkin-Ovchinnikov and vortex phases in a layered organic superconductor. J. Abbr. 2008, 10, 142-149. [CrossRef]

36. Pustogow, A.; Saito, Y.; Rohwer, A.; Schlueter, J.A.; Dressel, M. Coexistence of charge order and superconductivity in organic superconductor $\beta^{\prime \prime}$-(BEDT-TTF $)_{2} \mathrm{SF}_{5} \mathrm{CH}_{2} \mathrm{CF}_{2} \mathrm{SO}_{3}$. Phys. Rev. B 2019, 99, 140509(R). [CrossRef]

37. Shimahara, H. Structure of the Fulde-Ferrell-Larkin-Ovchinnikov state in two-dimensional superconductors. J. Phys. Soc. Jpn. 1998, 67, 736-739. [CrossRef]

38. Geiser, U.; Schlueter, J.A.; Wang, H.H.; Kini, A.M.; Williams, J.M.; Sche, P.P.; Zakowicz, I.H.; Michael, V.; Dudek, D.J.; Nixon, P.G.; et al. Superconductivity at $5.2 \mathrm{~K}$ in an electron donor radical salt of bis (ethylenedithio) tetrathiafulvalene (BEDT-TTF) with the novel polyfluorinated organic anion $\mathrm{SF}_{5} \mathrm{CH}_{2} \mathrm{CF}_{2} \mathrm{SO}_{3}$. J. Am. Chem. Soc. 1996, 118, 9996-9997. [CrossRef]

39. Yasuzuka, S.; Uji, S.; Terashima, T.; Sugii, K.; Isono, T.; Iida, Y.; Schlueter, J.A. In-Plane Anisotropy of Upper Critical Field and Flux-Flow Resistivity in Layered Organic Superconductor $\beta^{\prime \prime}$-(BEDT-TTF) ${ }_{2} \mathrm{SF}_{5} \mathrm{CH}_{2} \mathrm{CF}_{2} \mathrm{SO}_{3}$. J. Phys. Soc. Jpn. 2015, 84, 094709. [CrossRef]

40. Mori, T.; Kobayashi, A.; Sasaki, Y.; Kobayashi, H.; Saito, G.; Inokuchi, H. The Intermolecular Interaction of Tetrathiafulvalene and Bis(ethylenedithio)tetrathiafulvalene in Organic Metals. Calculation of Orbital Overlaps and Models of Energy-band Structures. Bull. Chem. Soc. Jpn. 1984, 57, 627. [CrossRef]

41. Beckmann, D.; Wanka, S.; Wosnitza, J.; Schlueter, J.A.; Williams, J.M.; Nixon, P.G.; Winter, R.W.; Gard, G.L.; Ren, J.; Whangbo, M.-H. Characterization of the Fermi surface of the organic superconductor-by measurements of Shubnikov-de Haas and angle-dependent magnetoresistance oscillations and by electronic band-structure calculations. Eur. Phys. J. Condens. Matter Complex Syst. 1998, 3, 295-300. [CrossRef]

42. Wosnitza, J.; Wanka, S.; Hagel, J.; Häussler, R.; Löhneysen, H.V.; Schlueter, J.A.; Geiser, U.; Nixon, P.G.; Winter, R.W.; Gard, G.L. Shubnikov-de Haas effect in the superconducting state of an organic superconductor. Phys. Rev. B 2000, 62, R11973. [CrossRef]

43. Wosnitza, J.; Hagel, J.; Meeson, P.J.; Bintley, D.; Schlueter, J.A.; Mohtasham, J.; Winter, R.W.; Gard, G.L. Enhanced magnetic quantum oscillations in the mixed state of a two-dimensional organic superconductor. Phys. Rev. B 2003, 67, 060504. [CrossRef]

44. Sugiura, S.; Terashima, T.; Uji, S.; Schlueter, J.A. Deformed Waveshape of Quantum Oscillation in Magnetocaloric Effect for Layered Organic Superconductor. J. Phys. Soc. Jpn. 2021, 90, 074601. [CrossRef]

45. Croitoru, M.D.; Houzet, M.; Buzdin, A.I. In-Plane Magnetic Field Anisotropy of the Fulde-Ferrell-Larkin-Ovchinnikov State in Layered Superconductors. Phys. Rev. Lett. 2012, 108, 207005. [CrossRef] [PubMed] 